DOI: https://doi.org/10.47405/mjssh.v5i9.474

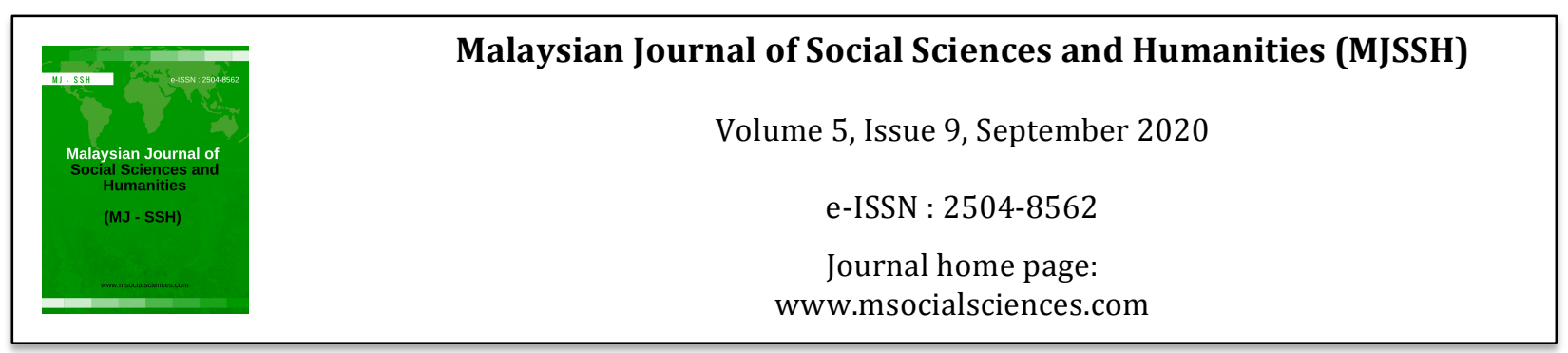

\title{
The Relationship Between Suicidal Ideation and Cognitive Distortion Among Undergraduates
}

\author{
Tan Yao Xiong1, Amalia Madihie', Jamayah Saili¹, Mohamad Azhari Abu Bakar¹, Rizal Abu Bakar1, \\ Mohd Izwan Mahmud² \\ 1Faculty of Cognitive Sciences and Human Development, Universiti Malaysia Sarawak (UNIMAS) \\ ${ }^{2}$ Faculty of Education, Universiti Kebangsaan Malaysia (UKM) \\ Correspondence: Tan Yao Xiong (yaoxiong@ukm.edu.my)
}

\begin{abstract}
Suicide has become a debilitating issue. Although it is a preventable condition but failure to understand the thinking pattern could significantly dampen the effort to avert it. Despite being an important issue, there is still a lack of study centralising into the thinking pattern when suicidal thoughts are mooted in. Due to this gap, this study was set to examine the relationship between cognitive distortion and suicidal ideation. Using purposive sampling 414 students whose were seeking career advice were recruited to participate in this study. Results indicated that there was a significant relationship between cognitive distortion and suicidal ideation. Using multiple regression, the finding showed that out of 10 thinking patterns, overgeneralisation contributes the most to the suicidal thoughts. These findings will help professionals to understand better the nature of suicidal ideation and assist them in providing better services and alleviating its symptoms. This study also discussed the limitations and the directions for future research.
\end{abstract}

Keywords: suicidal ideation, thinking pattern, cognitive distortion, catastrophizing, overgeneralization

\section{Introduction}

Suicide has become a debilitating issue that happens globally. It is not only affecting individuals adversely, but also causing destructive impacts to the society. For instance, almost 500 million individuals suffer from bereavement due to loss of significant individuals who have committed suicide annually (Pitman, Osborn, King, \& Erlangsen, 2014). According to World Health Organization (WHO) (2018), suicide has been classified as the $17^{\text {th }}$ cause of death among general population, and specifically the $2^{\text {nd }}$ main cause of death among individuals with the age range from 15 to 29 years old. Although the global crude suicide rate had been decreased approximately $12.4 \%$ across 15 years (2000: 12.2 to 2015:10.7), the rate was still relatively static and worrying. Unlike global rate, the crude suicide rate in Malaysia had been increased around 3.8\% from 2000 (5.3) to 2015 (5.5), and is expected to have continuous growth if no appropriate preventive interventions would be implemented (Malaysian Digest, 2015; WHO, 2018).

Suicide is an intended action that leads to death. It is basically initiated by the formation of suicidal ideation, which is an uncommon suicidal beliefs ranged from fleeting thoughts, extensive thoughts, comprehensive planning until incomplete suicide attempts (Choi, Lee, Yoon, Won, \& Kim, 2017; 
Haile et al., 2018). Commonly, individuals with suicidal ideation share certain physical and psychological symptoms, such as changing eat and sleep habits, having the senses of helplessness and hopelessness, declining in self-esteem and concentration, and experiencing emotional instability and racing thoughts. Suicidal ideation is believed to be significantly related to depression, which is known as a range of mood disturbance experienced by an individual varying from mild to severe and from transient to persistent (Preveler et al., 2002). This assumption had been proved by numerous previous researches, who proposed that individuals with higher level of depression would be more likely to develop higher level of suicidal ideation, eventually lead to higher chance of committing complete suicide (Liu, Zhang, Hennessy, Zhao, \& Ji, 2019; Medrano, Rosales, \& Gamez-Guadix, 2018; Rogers et al., 2018). Besides that, numerous researches had been performed to study the relationship between suicidal ideation and other mental illnesses, such as anxiety, posttraumatic stress disorder (PTSD), schizophrenia, etc., and the researchers proved that individuals with mental illnesses are more vulnerable to suicidal ideation (Panagioti, Gooding, \& Tarrier, 2012; Stanley et al., 2018). However, the reviewed correlational studies did not study the underlying thinking patterns that cause individuals developing suicidal ideation.

Cognitive distortions are known as several problematic thinking processes that adversely impact individuals' ability to perceive reality positively (Gross, 2015). Selective abstraction, dichotomous thinking, arbitrary inference, magnification or minimization, overgeneralization, and personalization are the first six types of cognitive distortions introduced by Aaron T. Beck (Roberts, 2015). Then, Burns have proposed new cognitive distortions, such as discounting the positive, emotional reasoning, and should statements. He also redefined and renamed terms "selective abstraction" and "arbitrary inference" to "mental filter" and "jumping to conclusions". After that, Freeman and DeWolf proposed three new cognitive distortions, which are externalization of self-worth, perfectionism, and comparison to others (Roberts, 2015). These distorted thoughts can cause individuals perceiving destructively on the world and experiencing depression and anxiety during undesirable conditions. Consequently, individuals would develop suicidal ideation (Gross, 2015). In order to conceptualize this explanation, the CBT Model of Suicide was constructed to understand the suicide among depressed individuals (Matthews, 2013). Based on Matthews (2013), several cognitive distortions, such as negative views of self, others, and future, dichotomous thinking, cognitive rigidity, attentional bias, attentional fixation and overgeneralized memory, are the crucial factors that affect depressed individuals experiencing impaired problem-solving ability and hopelessness, eventually lead to suicide.

\section{Literature Review}

Several researchers were also initiated to study the mechanisms between cognitive distortion and suicidal ideation. For instance, Jager-Hyman et al. (2014) investigated the relationship between suicide attempts and cognitive distortion among 168 patients (control group: 57 hospitalized patients without suicide attempts; uncontrolled group: 111 patients with suicide attempts). The finding showed that there were significant differences in term of cognitive distortion, depression, and hopelessness between control and uncontrolled group. The score of cognitive distortion was higher among samples with suicide attempts. The finding also proved that cognitive distortion (i.e., hopelessness and fortune telling) was the predictive factor of suicide. However, lack of reliability of certain variables in ICD (i.e., perfectionism), no diagnostic interview during sample selection, and absence of historical screening on suicide attempts among samples in control groups were the limitations of this study. The result obtained by Jager-Hyman et al. (2014) was inconsistent to the result of Khan, Upmanyu, Vinayak, and Kumar (2014), who also investigated the relationship between suicidal ideation and cognitive rigidity (a form of cognitive distortion). The researchers chose male adolescents $(n=150)$ with an age range from 15 to 17 years old from several government schools at Chandigarh as the samples. The researchers reported that suicidal ideation was not significantly related to cognitive rigidity (Khan et al., 2014). The acquired finding would not be sufficiently generalized the entire population as only small sample size from one racial group and gender was included in this study. Moreover, Wolff et al. (2014) conducted a cross-sectional study to investigate the relationship among victimization, cognitive distortion (i.e. negative self-talk and cognitive errors), perceived social 
support, and suicidal ideation. The psychiatrically hospitalized adolescents $(n=183)$ had been randomly selected as the samples. The finding proved that there was positive relationship between suicidal ideation with victimization and cognitive distortion, and negative relationship with perceived social support (Wolff et al., 2014). However, the selected samples were failed to representatively generalize the entire population as all samples were from similar ethnic group. Furthermore, FazakasDeHoog, Rnic, and Dozois (2017) studied the mechanism between suicidal ideation and cognitive distortions in order to design an integrative model of cognitive distortion and deficits. This research included a total of 397 undergraduates, and it proposed that cognitive distortion was the only variable that affects the establishment of suicidal ideation (Fazakas-DeHoog et al., 2017). Although big sample size was included, the appropriate data screening and mining were not performed during the process of selecting samples from the individuals with and without suicidal ideation. Thus, the result was unable to concisely represent the population. Additionally, the variables included in this study were not comprehensively represented the aspects of cognitive distortion and deficits.

From the previous studies, the research findings about the relationship between cognitive distortion and suicidal ideation were inconsistent. Theoretically, cognitive distortion is the initial factor causing development of suicidal ideation. This was supported by the studies conducted by Fazakas-DeHoog et al. (2017), Jager-Hyman et al. (2014), and Wolff et al. (2014), who proposed that cognitive distortion was significantly related to suicidal ideation. However, these findings were contradicted to the result of Khan et al. (2014) who found that there was no significant relationship between cognitive distortion and suicidal ideation. Additionally, the study related to the association between suicidal ideation and cognitive distortion is still lacking, especially the research that includes all types of cognitive distortion. Moreover, limited researches had been done in Asian. Thus, these results were not representatively generalized for the Asian population. Furthermore, most of the previous researches selected patients with suicidal ideation or other psychological disorders, but limited researches studying the population of adolescents and young adults who are aged from 15 to 29 years have been performed even though WHO (2018) had proposed that suicide is the $2^{\text {nd }}$ leading cause of death among this population. Therefore, the objectives of this study were to study the relationship between cognitive distortion and suicidal ideation and investigate the prediction of suicidal ideation by all subtypes of cognitive distortion among undergraduates who have received career advices in UKM.

\section{Methods}

This research used quantitative method to study the relationship between cognitive distortion and suicidal ideation. The targeted population $(N=1,000)$ in this study was the undergraduates (15 to 29 years old) who have received career advice at Universiti Kebangsaan Malaysia (UKM). Based on the formula proposed by Krejcie and Morgan (1997), the sample size was $n=278$. Purposive sampling was used to recruit samples. In this study, a total of 437 undergraduates had been selected as samples. Besides that, two established psychological instruments had been selected in this study, namely Beck Scale for Suicidal Ideation (BSS) and Cognitive Distortion Scale (CDS). BSS was an instrument developed by Aaron T, Beck, which can be used to assess the intensity of suicidal ideation (Rush, First, \& Blacker, 2008). It is a self-administered questionnaire made up of 21 items in which the first 19 items are used to assess the severity of suicidal ideation and the last two items are used to obtain information of suicide attempts. It used 3-point Likert scale (0 to 2). On the other hands, CDS is a selfadministered instrument that is used to assess 10 types of cognitive distortion, including mindreading, mental filter, personalization, catastrophizing, overgeneralization, all-or-nothing thinking, disqualifying the positive, emotional reasoning, should statements and labelling (Rnic, Dozois, \& Martin, 2016). Unlike BSS, CDS consists of 20 items and uses 7-point Likert-scale (1 to 7). CDS and BSS have desirable validity and reliability as both instruments were designed and adapted from previous studies.

The obtained raw data $(n=437)$ had been keyed in by using Statistical Package for Social Science (SPSS for Microsoft Windows) version 22.0. The process of data screening based on Pallant (2005), such as checking for errors, finding the error in the data file, and correcting the error in the data file, had been conducted. Then, Missing Value Analysis was performed to identify the presence of missing 
value, and the missing values $(n=17)$ had been removed. Additionally, the normality test was conducted, and the results of Kolmogorov-Smirnov statistic was reviewed as $n>50$ (Pallant, 2005). Furthermore, the process of checking for outlier was conducted, the result showed the presence of outliers $(n=6)$, which would possibly lead to substantial distortions of parameter and statistic estimates as well as inflated error rates when performing both parametric and nonparametric tests (Osborne \& Overbay, 2004). As the variables in this research were highly correlated with others, and it was impossible to change the shape of curve to be more normally distributed, the outliers had been removed (Tabachnick \& Fidell, 2013). Finally, the statistical methods, including normality test, frequency analysis, Pearson's Product-Moment Correlation and Multiple Regression were performed to test the hypothesis.

\section{Results}

\section{Descriptive Statistics}

After screening process, a total of 414 samples had been included in this study. The percentages of male samples were $23.4 \%(n=15)$ and female samples were $76.6 .5 \%(n=76)($ refer Table 1$)$. Table 2 showed the percentage of samples based on race. The race with highest percentage was Malay, which was $77.3 \%(n=320)$, whereby the lowest percentage was other races that are not stated in this questionnaire, which was $0.7 \%(n=3)$. Besides that, the percentages of Chinese, Indian, native of Sabah and native of Sarawak were 13.0\% $(n=54), 5.8 \%(n=24), 2.2 \%(n=9)$ and $1.0 \%(n=4)$.

Table 1: The descriptive statistic of samples based on gender

\begin{tabular}{lcc}
\hline Race & Frequency & Percent $\mathbf{( \% )}$ \\
\hline Male & 97 & 16.5 \\
Female & 317 & 83.5 \\
Total & 414 & 100.0 \\
\hline
\end{tabular}

Table 2: The descriptive statistic of samples based on race

\begin{tabular}{lcc}
\hline Race & Frequency & Percent (\%) \\
Malay & 320 & 77.3 \\
Chinese & 54 & 13.0 \\
India & 24 & 5.8 \\
Native of Sarawak & 4 & 1.0 \\
Native of Sabah & 9 & 2.2 \\
Others & 3 & .7 \\
Total & 414 & 100.0 \\
\hline
\end{tabular}

The descriptive analyses of suicidal ideation and cognitive distortions have been shown in Table 3 . The mean score of suicidal ideation was $4.36(S D=5.39)$, which means that the suicidal ideation of samples was low. Additionally, the mean score of cognitive distortions was $79(S D=22.4)$. Moreover, all types of cognitive distortion were descriptively analysed, including mindreading $(M=9.17, S D=$ $2.89)$, emotional reasoning $(M=8.31, S D=3.00)$, catastrophizing $(M=8.23, S D=2.97)$, all-or-nothing thinking $(M=8.03, S D=2.88)$, should statements $(M=7.97, S D=3.08)$, personalization $(M=7.87$, $S D=2.81)$, labelling $(M=7.71, S D=3.27)$, minimizing or disqualifying the positive $(M=7.52, S D=$ $3.08)$, mental filter $(M=7.34, S D=3.11)$ and overgeneralization $(M=6.87, S D=3.24)$. From Table 4 , mindreading was the cognitive distortion with highest mean score, whereas overgeneralization possessed lowest mean score. 
DOI: https://doi.org/10.47405/mjssh.v5i9.474

Table 3: The mean scores and standard deviations of variables.

\begin{tabular}{lccccc}
\hline Variables & N & Minimum & Maximum & Mean & Std. Deviation \\
\hline Suicidal Ideation & 414 & 0 & 24 & 4.36 & 5.39 \\
Cognitive Distortions & 414 & 20 & 134 & 79.0 & 22.4 \\
\hline
\end{tabular}

Table 4: The mean scores and standard deviations of all types of cognitive distortion

\begin{tabular}{lccccc}
\hline Cognitive Distortions & N & Minimum & Maximum & Mean & Std. Deviation \\
\hline Mindreading & 414 & 2 & 14 & 9.17 & 2.89 \\
Emotional Reasoning & 414 & 2 & 14 & 8.31 & 3.00 \\
Catastrophizing & 414 & 2 & 14 & 8.23 & 2.97 \\
All-or-nothing Thinking & 414 & 2 & 14 & 8.03 & 2.88 \\
Should Statements & 414 & 2 & 14 & 7.97 & 3.08 \\
Personalization & 414 & 2 & 14 & 7.87 & 2.81 \\
Labelling & 414 & 2 & 14 & 7.71 & 3.27 \\
Minimizing or & 414 & 2 & 14 & 7.52 & 3.08 \\
Disqualifying the Positive & & & & & \\
Mental Filter & 414 & 2 & 14 & 7.34 & 3.11 \\
Overgeneralization & 414 & 2 & 14 & 6.87 & 3.24 \\
\hline
\end{tabular}

\section{Hypotheses Testing}

All hypotheses of this research were tested as follows:

\section{Ho1: There is no significant relationship between suicidal ideation and cognitive distortions among undergraduates who have received career advices in UKM.}

The result showed that suicidal ideation was positively and significantly related to cognitive distortion $(r(412)=.46, p<.000)$, as its $\mathrm{p}$ value was smaller than .05 (refer to Table 5). Thus, the null hypothesis was rejected.

Table 5: The correlations between suicidal ideation and cognitive distortions

\begin{tabular}{llll}
\hline & & Sum_SI & Sum_CD \\
\hline \multirow{3}{*}{ Sum_SI } & Pearson Correlation & 1 & $.46^{* *}$ \\
& Sig. (2-tailed) & & .000 \\
${ }^{*} \mathrm{p}<.05, * * \mathrm{p}<.01, * * * \mathrm{p}<.001$ & 414 & 414
\end{tabular}

$H_{0}$ : There is no significant relationship between suicidal ideation with all types of cognitive distortions among undergraduates who have received career advices in UKM.

There was a positive significant relationship between suicidal ideation with all types of cognitive distortions, including labelling $(r(412)=.39, p=.000)$, mental filter $(r(412)=.38, p=.000)$, overgeneralization $(r(412)=.38, p=.000)$, personalization $(r(412)=.37, p=.000)$, catastrophizing $(r(412)=.35, p=.000)$, emotional reasoning $(r(412)=.34, p=.000)$, minimizing or disqualifying the positive $(r(412)=.32, p=.000)$, mindreading $(r(412)=.30, p=.000)$, should statements $(r(412)=$ $.27, p=.000)$ and all-or-nothing thinking $(r(412)=.26, p=.000)$. From Table 6 , all $p$ values were smaller than .05 . Thus, the null hypothesis was rejected. 
DOI: https://doi.org/10.47405/mjssh.v5i9.474

Table 6: The correlations between suicidal ideation and all types of cognitive distortions

\begin{tabular}{cccccccccccc}
\hline & $\begin{array}{c}\text { Sum } \\
\text { SI }\end{array}$ & MR & C & ANT & ER & L & ME & OG & P & SS & MDP \\
\hline$p$ & 1 & $.30^{* *}$ & $.35^{* *}$ & $.266^{* *}$ & $.34^{* *}$ & $.39^{* *}$ & $.38^{* *}$ & $.38^{* *}$ & $.37^{* *}$ & $.27^{* *}$ & $.32^{* *}$ \\
& & & & & & & & & & & \\
& & .000 & .000 & .000 & .000 & .000 & .000 & .000 & .000 & .000 & .000 \\
$\mathrm{~N}$ & 414 & 414 & 414 & 414 & 414 & 414 & 414 & 414 & 414 & 414 & 414 \\
\hline
\end{tabular}

$* \mathrm{p}<.05, * * \mathrm{p}<.01, * * * \mathrm{p}<.001$

MR $=$ Mindreading

$\mathrm{C}=$ Catastrophizing

ANT = All-or-nothing thinking

ER = Emotional reasoning

L $\quad=$ Labelling

ME $=$ Mental Filter

OG = Overgeneralization

$\mathrm{P} \quad=$ Personalization

SS $\quad=$ Should statements

MDP = Minimizing or disqualifying the positive

\section{Ho3: There is no significant prediction of suicidal ideation by all types of cognitive distortions among undergraduates who have received career advice in UKM.}

Table 7 and 8 illustrated that a significant regression equation was found $(\mathrm{F}(10,403)=11.77, p=$ $.000)$, with an $\mathrm{R}^{2}$ of .23 . Among all types of cognitive distortions, only overgeneralization $(\beta=.14, p=$ .02 ) was the significant predictor of suicidal ideation as its $p$ value was lower than .05 (refer Table 9). Thus, the null hypothesis was rejected.

Table 7: The model summary that included suicidal ideation (DV) and all types of cognitive distortions (IV)

\begin{tabular}{ccccc}
\hline Model & R & R Square & Adjusted R Square & td. Error of the Estima \\
\hline 1 & $.48^{\text {a }}$ & .23 & .21 & 4.80 \\
\hline
\end{tabular}

Table 8: The result of ANOVA between regression and residual

\begin{tabular}{ccccccc}
\hline & Model & Sum of Squares & Df & Mean Square & F & Sig. \\
\hline \multirow{4}{*}{1} & Regression & 2713.3 & 10 & 271.3 & 11.77 & $.000^{\mathrm{b}}$ \\
& Residual & 9289.5 & 403 & 23.1 & & \\
& Total & 12002.8 & 413 & & & \\
\hline
\end{tabular}

Table 9: The standardized coefficients of regression

\begin{tabular}{lllll}
\hline Model & $\begin{array}{l}\text { Standardized } \\
\text { Coefficients } \\
\text { Beta }\end{array}$ & T & Sig. \\
\hline (Constant) & & -3.97 & .000 \\
Sum_Mindreading & .02 & .39 & .70 \\
& Sum_Catastrophizing & .08 & 1.32 & .19 \\
& Sum_Allornothingthinking & -.01 & -.11 & .91 \\
& Sum_Emotional_Reasoning & .03 & .51 & .61 \\
Sum_Labeling & .11 & 1.60 & .11 \\
Sum_Mental_Filter & .08 & 1.27 & .20 \\
Sum_Overgeneralization & .14 & 2.27 & .02
\end{tabular}


Malaysian Journal of Social Sciences and Humanities (MJSSH), Volume 5, Issue 9, (page 30 - 38), 2020

DOI: https://doi.org/10.47405/mjssh.v5i9.474

\begin{tabular}{llll} 
Sum_Personalization & .11 & 1.78 & .08 \\
Sum_Should_Statements & -.06 & -1.02 & .30 \\
Sum_Minimizing_Or_Disqualifying_The_Positive.09 & 1.69 & .09 \\
\hline
\end{tabular}

\section{Discussions}

The obtained results were consistent to the findings of previous studies conducted by Fazakas-DeHoog et al. (2017), Jager-Hyman et al. (2014), and Wolff et al. (2014), who proposed that cognitive distortion was significantly and positively related to suicidal ideation. This represented that individuals with cognitive distortions would be more likely to develop suicidal ideation. According to CBT, cognitive distortion is the maladaptive thinking pattern that affects individuals perceiving reality inappropriately, and it is greatly influenced by the presence of negative schemas. Schema is the mental structure of concepts or a system that manages and interprets raw information (Goldstein, 2011; Matthews, 2013). Based on Piaget's Theory of Cognitive Development, schema is distinctive and unique for each individual as it is formed based on personal experience and information obtained from the world (Wadsworth, 2004). The schemas are closely related to individuals' emotions and behaviours (Wadsworth, 2004). In this case, cognitive distortions are the results of dysfunctional schemas due to undesirable life events (i.e., traumatic experience, violence, bullying, parents' divorce, etc.) experienced by individuals since birth. Consequently, these thinking patterns caused individuals having emotional and behavioural disturbances during stressful and displeasing circumstances. Eventually, those individuals would develop suicidal ideation and attempts as they are unable to cope with psychological pains except death.

Additionally, based on Norman and Shallice model, the activation of schema is regulated by two mechanisms of executive functions, namely contention scheduling and supervisory attentional system (SAS) (Friedenberg \& Silverman, 2010). These are the cognitive apparatuses that play role in the regulation and management of cognitive processes. Contention scheduling monitors the activation of schema when experiencing routine and well-learned circumstances, whereas SAS activates schema under non-routine and distinct conditions (Friedenberg \& Silverman, 2010). Both systems are performed and regulated by frontal lobes, which is the crucial part of brain that performs higher cognitive processes, working memory and executive functioning including decision making, executive functioning problem-solving, attention, etc. (Goldstein, 2011; Sabir \& Khalily, 2016). Sabir and Khalily (2016) also proposed that temporal lobe was an additional part that plays role in storing and controlling information in long terms. Therefore, damage to or unhealthy brain development, especially these two lobes, would adversely disturb the activation and functions of schemas. Subsequently, those individuals possess distorted thoughts, eventually lead to suicidal ideation.

Although all types of cognitive distortions were significantly related to suicidal ideation among undergraduates who looked for career advices, their relational coefficients were generally low $(<.40)$. Besides that, overgeneralization was the only cognitive distortions that significantly predicted and explained suicidal ideation. According to Gross (2015), overgeneralization is a cognitive error in which individuals make generalized conclusions according to a single experience. In this case, individuals would draw conclusions that suicide is the only method to terminate the psychological pain according to pass life experience. Thus, restructuring of these dysfunctional thinking patterns is an utmost important aspect to be highlighted in the process of intervention with the aim of helping that particular individual having capability to thinking in a more constructive and positive way (Clark \& Beck, 2010).

\section{Implications and Recommendation}

From the findings, this research contributed to the current understanding of suicide from cognitive perspective. This information will also help mental health professionals to conceptualize better the nature of suicidal ideation and assist them in providing better services and alleviating the symptoms of suicide. The findings also acted as the theoretical platform for designing and implementing appropriate 
DOI: https://doi.org/10.47405/mjssh.v5i9.474

interventions. Despite implications, several limitations had been found in this study. For instance, the independent variables included in this research were not comprehensive enough to explain suicidal ideation as all cognitive distortions only explained $22.6 \%$ of suicidal ideation. Additionally, the samples were not randomly selected from each faculty, which caused targeted population may not be representatively generalized the entire population. Therefore, the researchers were recommended to include other cognitive factors and different types of cognitive distortions, including perfectionism, externalization of self-worth, and comparison to others. The future researchers were also suggested using random sampling to select sample and conducting study among individuals with suicidal ideations or attempts.

\section{Conclusion}

In conclusion, this research aimed to study the relationship between cognitive distortions and suicidal ideation among undergraduates who received career advice in UKM. The result showed that there was a significant positive relationship between cognitive distortions and suicidal ideation. Additionally, all types of cognitive distortions were significantly and positively associated with suicidal ideation and overgeneralization was the only cognitive distortion that contributed most to the development of suicidal ideation. The limitations and recommendation for future study had been proposed.

\section{Acknowledgement}

This study is funded by the National University of Malaysia GG-2019-078. Funding in this paper is part of a research grant.

\section{References}

Choi, S. B., Lee, W., Yoon, J. H., Won, J. U., \& Kim, D. W. (2017). Risk factors of suicide attempt among people with suicidal ideation in South Korea: A cross-sectional study. BMC Public Health, 17(1), 579-568.

Clark, D. A., \& Beck, A. T. (2010). Cognitive theory and therapy of anxiety and depression: Convergence with neurobiological findings. Trends in Cognitive Sciences, 14(9), 418-424.

Fazakas-DeHoog, L. L., Rnic, K., \& Dozois, D. J. (2017). A cognitive distortions and deficits model of suicide ideation. Europe's Journal of Psychology, 13(2), 178-193.

Friedenberg, J., \& Silverman, G. (2010). Cognitive science: An introduction to the study of mind. Thousand Oaks, California: Sage Publications, Inc.

Goldstein, E. B. (2011). Cognitive psychology: Connecting mind, reserch, and everday expereince (3rd ed.). Wadsworth: Cengage Learning.

Gross, R. (2015). Psychology: The science of mind and behaviour (7th ed.). United Kingdom: Hodder Education.

Haile, K., Awoke, T., Ayano, G., Tareke, M., Abate, A., \& Nega, M. (2018). Suicidal ideation and attempts among people with epilepsy in Addis Ababa, Ethiopia. Annals of General Psychiatry, 17(4), 1-8.

Jager-Hyman, S., Cunningham, A., Wenzel, A., Mattei, S., Brown, G. K., \& Beck, A. T. (2014). Cognitive distortions and suicide attempts. Cognitive Therapy and Research, 38(4), 369-374.

Khan, I., Upmanyu, V. V., Vinayak, S., \& Kumar, S. (2014). Study on depression, hopelessness, anxiety, cognitive rigidity, affective dysregulation and family environment as predictors of suicide ideation among male adolescents. Indian Journal of Health \& Wellbeing, 5(10), 11241129.

Krejcie, R. V., \& Morgan, D. W. (1970). Determining sample size for research activities. Educational and Psychological Measurement, 30, 607-610.

Liu, Y., Zhang, J., Hennessy, D. A., Zhao, S., \& Ji, H. (2019). Psychological strains, depressive symptoms, and suicidal ideation among medical and non-medical staff in urban China. Journal of Affective Disorders, 245(3), 22-27. 
Malaysia Digest. (2015). Suicide is the 2nd leading cause of death among youth: A closer look at the situation in Malaysia. Retrieved from http://www.malaysiandigest.com/features/568013suicide-is-the-2nd-leading-cause-of-death-among-youth-a-closer-look-at-the-situation-inmalaysia.html

Matthews, J. D. (2013). Cognitive Behavioral Therapy approach for suicidal thinking and behaviors in depression. Retrieved from https://www.intechopen.com/books/mental-disorders-theoreticaland-empirical-perspectives/cognitive-behavioral-therapy-approach-for-suicidal-thinking-andbehaviors-in-depression

Medrano, J. L. J., Rosales, F. L., \& Gamez-Guadix, M. (2018). Assessing the links of sexting, cybervictimization, depression, and suicidal ideation among university students. Archives of Suicide Research, 22(1), 153-164.

Osborne, J. W., \& Overbay, A. (2004). The power of outliers (and why researchers should always check for them). Practical Assessment, Research \& Evaluation, 9(6), 1-12.

Pallant, J. (2005). SPSS survival manual: A step by step guide to data analysis using SPSS for Windows (version 12). Crows Nest NSW, Australia: Allen \& Unwin.

Panagioti, M., Gooding, P. A., \& Tarrier, N. (2012). A meta-analysis of the association between posttraumatic stress disorder and suicidality: The role of comorbid depression. Comprehensive Psychiatry, 53(7), 915-930.

Peveler, R., Carson, A., \& Rodin, G. (2002). ABC of psychological medicine: Depression in medical patients. British Medical Journal, 325(7356), 149-152.

Pitman, A., Osborn, D., King, M., \& Erlangsen, A. (2014). Effects of suicide bereavement on mental health and suicide risk. The Lancet Psychiatry, 1(1), 86-94.

Rnic, K., Dozois, D. J., \& Martin, R. A. (2016). Cognitive distortions, humor styles, and depression. Europe's Journal of Psychology, 12(3), 348-362.

Rogers, M. L., Stanley, I. H., Hom, M. A., Chiurliza, B., Podlogar, M. C., \& Joiner, T. E. (2018). Effects of suicide bereavement on mental health and suicide risk. Assessment, 25(2), 159-172.

Rush, J. A., First, M. B., \& Blacker, D. (2008). Beck Scale for Suicide Ideation (BSS). In A. Beck, M. Kovacs, \& A. Weissman, Handbook of psychiatric measures (2nd ed.) (pp. 242-244). Washington, DC:: American Psychiatric Publishing, Inc.

Sabir, Z., \& Khalily, M. T. (2016). Executive functioning and dysfunctional schema modes in individuals with frontal lobe lesion and temporal lobe epilepsy: Mega case analysis. Journal of Psychology and Clinical Psychiatry, 5(1), 246-251.

Stanely, I. H., Boffa, J. W., Rogers, M. L., Hom, M. A., Albanese, B. J., Chu, C.,...Joiner, T. E. (2018). Anxiety sensitivity and suicidal ideation/suicidal risk: A meta-analysis. Journal of Consulting and Clinical Psychology, 86(11), 946-960.

Tabachnick, B. G., \& Fidell, L. S. (2013). Using multivariate statistics (6th ed.). Upper Saddle River, New Jersey: Pearson Education, Inc..

Wadsworth, B. J. (2004). Piaget's theory of cognitive and affective development: Foundations of constructivism. United States: Longman Publishing.

Wolff, J., Esposito-Smythers, C., Becker, S., Seaboyer, L., Rizzo, C., Lichtenstein, D., \& Spirito, A. (2014). Social-cognitive moderators of the relationship between peer victimization and suicidal ideation among psychiatrically hospitalized adolescents. Journal of Aggression, Maltreatment \& Trauma, 23(3), 268-285.

World Health Organization. (2018). World health statistics data visualizations dashboard. Retrieved from http://apps.who.int/gho/data/view.sdg.3-4-data-ctry?lang=en 